\title{
Dynamic Evaluation and Convergence Analysis of the Efficiency of Rural Medical Health Centers in China
}

\section{Yun Ye}

School of Economics and Management, Yangtze University

\section{Richard Evans}

College of Engineering, Design and Physical Sciences, Brunel University London

\section{Shaoqiong Li}

Center for Information, Chinese Center for Disease Control and Prevention

Wei Lu

Department of Management ,Hainan Medical University

\section{Xudong Shang}

Center of Modern Agriculture, AMI of MARA

\section{Li Jing (D1581176010@qq.com )}

Department of Health Management, School of Medicine and Health Management, Tongji Medical College, Huazhong University of Science and Technology

\section{Research Article}

Keywords: Rural medical health centers, Efficiency, Convergence

Posted Date: January 18th, 2021

DOl: https://doi.org/10.21203/rs.3.rs-130813/v1

License: (c) (i) This work is licensed under a Creative Commons Attribution 4.0 International License. Read Full License 


\title{
Dynamic evaluation and convergence analysis of the efficiency of rural medical health centers in China
}

\author{
Yun $\mathrm{Ye}^{1}$, Richard Evans ${ }^{2}$, Shaoqiong $\mathrm{Li}^{3}$,Wei Lu ${ }^{4}$,Xudong Shang ${ }^{5}$, Li Jing ${ }^{6 *}$ \\ 1.School of Economics and Management, Yangtze University, Jingzhou, Hubei Province, \\ China; yeyun@yangtzeu.edu.cn \\ 2.College of Engineering, Design and Physical Sciences, Brunel University London, London, \\ UK; richard.evans@brunel.ac.uk \\ 3.Center for Information, Chinese Center for Disease Control and Prevention, Beijing, China; \\ lisq@chinacdc.cn \\ 4.Department of Management ,Hainan Medical University, Haikou, Hainan Province, China; \\ hy0205044@hainmc.edu.cn
}

5. Center of Modern Agriculture, AMI of MARA, Beijing,China; cauleone@126.com

6.Department of Health Management, School of Medicine and Health Management, Tongji

Medical College, Huazhong University of Science and Technology, Wuhan, Hubei Province,

China; 1581176010@qq.com

Correspondence:

Li Jing, Department of Health Management, School of Medicine and Health Management, Tongji Medical College, Huazhong University of Science and Technology, Wuhan, Hubei Province, China. Email: 1581176010@qq.com

Funding information: National Social Science Fund (17BZZ026)

\begin{abstract}
Background: Rural medical health centers (RMHCs) are the foundation of the three-level primary medical and health service system in China. The efficiency of RMHCs is related to the rationality of the health care resource allocation for China's
\end{abstract}


560 million rural population.

Methods: This study analyzed the dynamic changes in the efficiency of RMHCs and its convergence using the non-guided SBM-DEA window model and convergence model, in Shanxi Province, China.

Results: The results showed that the medical service efficiency is low. The average scores of the comprehensive technical efficiency and pure technical efficiency of the RMHCs in China from 2016 to 2018 were only 0.1541 and 0.1670 , respectively, with nearly $63 \%$ of the values being lower than their average scores. The comprehensive technical efficiency and pure technical efficiency of the RMHCs in 2016-2018 exhibited a downtrend year on year. The convergence analysis results also showed that the current rural health clinic medical service efficiency had absolute convergence and conditional convergence. If appropriate policies are followed, the medical service efficiency of the RMHCs can be improved to reach its steady-state level.

Conclusions: The medical service efficiency of RMHCs remains low and may gradually decline. The government should promote the efficiency of medical service in RMHCs to reach their own optimal level by formulating corresponding medical and health resource allocation policies.

\section{Keywords: Rural medical health centers, Efficiency, Convergence}

\section{Background}

China's health care reform was launched in 2009, and the medical service capacity of primary-level medical and health care institutions has been significantly improved. However, there remains an uneven distribution of health care resources between urban and rural areas ${ }^{[1]}$. With regard to this problem, allocating health care resources fairly is an important issue for all levels of government in China.

Rural medical health centers (RMHCs) are the foundation of China's three-tiered health service system, taking on important responsibilities for rural residents ${ }^{[2]}$. According to the China health statistics yearbook of 2019, 1.67 billion patients were 
diagnosed and treated in RMHCs, accounting for $20.13 \%$ of all outpatient visits in medical institutions at all levels across the country during 2018. Moreover, RMHCs take $40 \%$ of the responsibility of the basic public health services in China. The medical service efficiency score of RMHCs is related to the distribution of medical and health resources for the 560 million rural population of China ${ }^{[3]}$. Therefore, it is particularly important to evaluate the medical service efficiency of RMHCs and allocate medical and health resources reasonably to solve the existing problems in rural China.

Currently, some studies have evaluated the medical service efficiency of primary medical and health institutions. However, these studies mainly focused on county and town hospitals, with very few studies on $\mathrm{RMHCs}^{[4-7]}$. Therefore, this study takes RMHCs in Shanxi Province as the research object, and the data envelopment analysis (DEA) method is applied to measure their medical efficiency. The DEA method is a mature efficiency measurement method, which has been used by several scholars studying the medical service efficiency ${ }^{[8-11]}$. Moreover, we use the absolute and conditional convergences to analyze the convergence of RMHC efficiency. The convergence theory has been applied to the field of health care since 2000, and has been paid increasing attention by scholars since then. The convergence theory has been widely used in studying health service evaluation indices such as the total health cost, life expectancy, and mortality rate ${ }^{[12-14]}$. In this research, we apply the convergence analysis to answer whether the difference in the medical service efficiencies between RMHCs is gradually narrowing or expanding? Is the development of regions with a lower medical service efficiency lagging behind those with a higher medical service efficiency or surpassing the latter, which leads to the so-called convergence phenomenon?

In comparison with existing literature, the contributions of this paper are as follows: First, a dynamic evaluation of the medical service efficiency of China's RMHCs is carried out, and the true level and change trend in the current medical service efficiency of the RMHCs are obtained. Second, the convergence theory is 
applied to analyze the difference in the medical service efficiencies, so as to provide a basis for optimizing the dynamic management of rural medical and health resources in the future and promoting the rationality of health resource allocation.

\section{Methods}

\subsection{Data sources}

The data presented in this study were derived from the Shanxi Rural Health Institute's 2016-2018 Health Statistics Report, which involved 4834 RMHCs; patient information was not collected.

\subsection{Input and output variables}

Input and output variables are used in the DEA model, and the efficiency of the medical service is calculated using the input made by the hospitals and the output obtained from the input. Unlike county and town hospitals, RMHCs mainly provide rural residents with general diagnosis and treatment and referral medical services for common and frequently encountered diseases. Therefore, the input-output indicators of the RMHCs are quite different from those of county and town hospitals. We chose the number of rural doctors, technician training times for doctors, and drug expenditure as the input variables. The output variables were the number of patients and income from essential drugs.

\subsection{Data envelopment analysis model}

The DEA is a new field in operations research, management science, and mathematical economics. It is a quantitative analysis method to evaluate the relative effectiveness of comparable units of the same type by means of linear programming based on multiple input and output indices ${ }^{[15]}$. Conventional radial DEA models, such as the CCR and BCC models, only include the proportion of all inputs (outputs) reduced or increased. When evaluating an institution's efficiency, the influence of slack variables is not considered, which may result in the deviation of the efficiency measure. Tone ${ }^{[16]}$ proposed the SBM-DEA model (slack-based measure, as a function 
of the furthest distance to the frontier), which solved the problem that the radial model did not include the slack variable in the measurement of the inefficiency. The SBM-DEA model in this study is as follows:

$$
\begin{gathered}
\text { The objective function: } \min \rho=\frac{1-\frac{1}{m} \sum_{i=1}^{m} \frac{s_{i}^{-}}{x_{i k}}}{1+\frac{1}{q} \sum_{r=1}^{q} \frac{s_{r}^{+}}{y_{r k}}} \\
\text { Constraints: } x_{k}=x \lambda+s^{-}, y_{k}=y \lambda-s^{+}, \lambda, s^{-}, s^{+} \geq 0
\end{gathered}
$$

where $m$ and $q$ in the objective function respectively represent $m$ inputs and q outputs of each RMHC. $x_{i k}$ and $y_{r k}$ respectively represent the ith input and rth output of the kth RMHC. $s^{-}$and $s^{+}$respectively represent the slack variable of the ith input and the slack variable of the rth output. $\frac{s_{i}^{-}}{x_{i k}}$ and $\frac{s_{r}^{+}}{y_{r k}}$ respectively represent the inefficiencies of the ith input and rth output, respectively. In the constraints, $x_{k}$ and $y_{k}$ represent the input and output of the kth RMHC, respectively. $\lambda$ represents the adjustment matrix, $\mathrm{x} \lambda$ and $\mathrm{y} \lambda$ respectively represent the input and output of the frontier production line. $s^{-}$and $s^{+}$represent the slack variables of the input and output, respectively. The value of $\rho$ is between 0 and 1 . When $\rho=1$, it indicates that the RMHC is highly effective and located in the efficiency frontier, and each slack is 0 . When $\rho$ is close to 0 , it indicates that the RMHC is inefficient.

In addition, when using the SBM-DEA model to calculate the efficiency value, the calculated efficiency value is not comparable between different years, because the leading edge of each RMHC differs from year to year. The DEA window analysis method, proposed by Charnes ${ }^{[17]}$, is preferred to solve this type of problem. Its analysis principle involves treating institutions in different periods as different institutions and evaluating the relative efficiency of the institutions by selecting different reference sets similar to the moving average method. This method has two evident advantages: one is that the number of institutions in the reference set is multiplied several times, which is an effective way to solve the problem of insufficient number of institutions. Second, it can not only measure the efficiency of 
each institution on a section but also measure the change trend in the efficiency of all institutions in the time series. It reflects the change trend in the efficiency from a dynamic perspective by considering the relationship between different periods, hence the name dynamic DEA. In this study, the medical service efficiency refers to the comprehensive technical efficiency; comprehensive technical efficiency $=$ pure technical efficiency $\times$ scale efficiency.

In summary, the DEA model adopted in this study is the SBM-DEA model combined with the Window-DEA model to evaluate the efficiency of RMHCs in Shanxi Province, China.

\subsection{Convergence analysis}

There are three types of convergence analysis: $\alpha$ convergence, absolute $\beta$ convergence, and conditional $\beta$ convergence ${ }^{[18]}$. Notably, the $\beta$ convergence is a necessary but insufficient condition of the $\alpha$ convergence, because if there is an $\alpha$ convergence, the efficiency of the individual absolute gap in narrow, low efficiency of this type of situation only when individuals have higher than high level of efficiency of individual growth rate can be set up; however, individuals with $\beta$ convergence do not necessarily have $\alpha$ convergence.

In this study, the $\alpha$ convergence refers to the process whereby the medical service efficiency of RMHCs changes over time. The expression is:

$$
\sigma=\sqrt{\frac{1}{n} \sum_{i=1}^{n}\left(\ln T E_{i}-\frac{1}{n} \sum_{i=1}^{n} \ln T E_{i}\right)^{2}}
$$

where $\mathrm{T} E_{i}$ represents the medical service efficiency of the ith RMHC, and $n$ represents the total number of RMHCs. Based on the absolute $\beta$ convergence, the lower the medical service efficiency of the RMHCs, the higher the growth rate of the medical service efficiency. Therefore, as time passes, inefficient RMHCs will catch up with the efficient ones and reach a stable convergence state at the same speed. The expression of the absolute $\beta$ convergence is ${ }^{[19]}$. 


$$
\frac{\left[\ln T E_{i, t}-\ln T E_{i, 0}\right]}{T}=\alpha+\beta \ln T E_{i, 0}+\varepsilon_{i, 0}
$$

$\frac{\left[\operatorname{lnT} E_{i, t}-\ln T E_{i, 0}\right]}{T}$ represents the average annual growth rate of the medical service efficiency of the ith RMHC from the base period $\mathrm{t}=0$ to $\mathrm{t}=\mathrm{T}$. $\ln T E_{i, 0}$ represents the logarithm of the initial medical service efficiency score of the ith RMHC, $\beta$ is the convergence coefficient, and $\varepsilon_{i, 0}$ is the error term. If $\ln T E_{i, 0}$ is significant and negative, it indicates that the average annual growth rate of the medical service efficiency is negatively correlated with the efficiency score in the base period, indicating that there is a "catch-up effect" among RMHCs. In other words, the RMHCs with a low efficiency score will eventually have a higher growth rate, and the medical service efficiency will eventually approach the same steady-state level. On the contrary, if $\ln T E_{i, 0}$ is significant and positive, it indicates a widening gap in the medical service efficiency among RMHCs. The conditional $\beta$ convergence allows the RMHCs to have different characteristics, so that the gap between efficient and inefficient RMHCs persists. The expression of the conditional $\beta$ convergence is ${ }^{[20]}$ :

$$
\ln T E_{i, t}-\ln T E_{i, t-1}=\alpha+\beta \ln T E_{i, t-1}+\varepsilon_{i, t}
$$

In $\mathrm{T} E_{i, t}$ is the logarithm of the medical service efficiency score in the period $t$ of the ith RMHC, and $\beta$ is the convergence coefficient. If $\operatorname{lnT} E_{i, t-1}$ is significant and $\beta$ is negative, it indicates that the efficiency score of the ith RMHC will converge to its steady-state level over time.

\section{Results}

Table 1 shows the change trend in the input and output of the RMHCs from 2016 to 2018. In terms of the input variables, the number of rural doctors gradually declines from 2016 to 2018, the annual average number of rural doctors drops from 1.5308 in 2016 to 1.4727 in 2018, and the quantity gap between different RMHCs is significant. In 2018 , the number of rural doctors in some health clinics was only 1 , whereas in others, the number was as high as 15. From 2016 to 2018, the annual average number of technician training times for doctors showed an overall growth trend, from 13.6648 
in 2016 to 15.5985 in 2018. The drug expenditure showed a general downtrend from 32862.18 yuan in 2016 to 27998.08 yuan in 2018 , a decrease of nearly $15 \%$.

In terms of the output variables, from 2016 to 2018, the annual number of RMHC patients declined. In 2016, the average number of patients was 1630.17 , which declined to 1402.12 in 2018. During the same period, the average income from essential drugs also declined year on year, from 30779.68 yuan in 2016 to 25073.02 yuan in 2018. In addition, the drug expenditure and essential drugs income are important factors of RMHCs' revenues and expenditures. Although drug expenditure exhibited a downtrend from 2016 to 2018, it was still much higher than the income from essential drugs, indicating that the RMHCs needed to rely on government subsidies to maintain normal operations during this period.

Table 1 Summary statistic of input-output variables (2016-2018)

\begin{tabular}{|c|c|c|c|c|c|}
\hline & \multicolumn{3}{|c|}{ Input } & \multicolumn{2}{|c|}{ Output } \\
\hline & I1 & I2 & I3 & 01 & $\mathbf{O 2}$ \\
\hline \multicolumn{6}{|l|}{2016} \\
\hline Mean & 1.5308 & 13.6648 & 32862.18 & 1630.17 & 30779.68 \\
\hline Max & 15 & 879 & 620000 & 22000 & 620000 \\
\hline Min & 1 & 1 & 310 & 14 & 426 \\
\hline \multicolumn{6}{|l|}{2017} \\
\hline Mean & 1.5176 & 14.3014 & 33919.99 & 1642.45 & 31541.74 \\
\hline Max & 18 & 879 & 571866 & 28164 & 571866 \\
\hline Min & 1 & 1 & 658 & 10 & 484 \\
\hline \multicolumn{6}{|l|}{2018} \\
\hline Mean & 1.4727 & 15.5985 & 27998.08 & 1402.12 & 25073.02 \\
\hline
\end{tabular}




$\begin{array}{lllll}\text { Max } & 15 & 879 & 403019 & 12\end{array}$

$\begin{array}{llllll}\text { Min } & 1 & 1 & 100 & 25290 & 64\end{array}$

I1, number of rural doctors; I2, technician training times for doctors; I3, drug expenditure; O1, number of patients; $\mathrm{O} 2$, income from essential drugs.

Figure 1 shows the scores of the technical efficiency (TE), pure technical efficiency (PTE), and scale efficiency (SE) for the studied RMHCs in 2016-2018. In terms of the time series, the TE and PTE of the RMHCs declined year on year, whereas the SE remained generally stable. The average TE of RMHCs in 2016 was 0.1683 , which had decreased to 0.1304 in 2018 , a decrease of nearly $23 \%$. In the same period, the PTE decreased from 0.1820 in 2016 to 0.1433 in 2018, a decrease of nearly 22\%. The SE also declined slightly, from 0.9186 in 2016 to 0.9035 in 2018, only by $2 \%$. Therefore, the decline in the TE during 2016-2018 is mainly due to the decline in the PTE. At the present stage, the TE and PTE were both low, with nearly $63 \%$ of the TE and PTE values being lower than the average score of 2018.

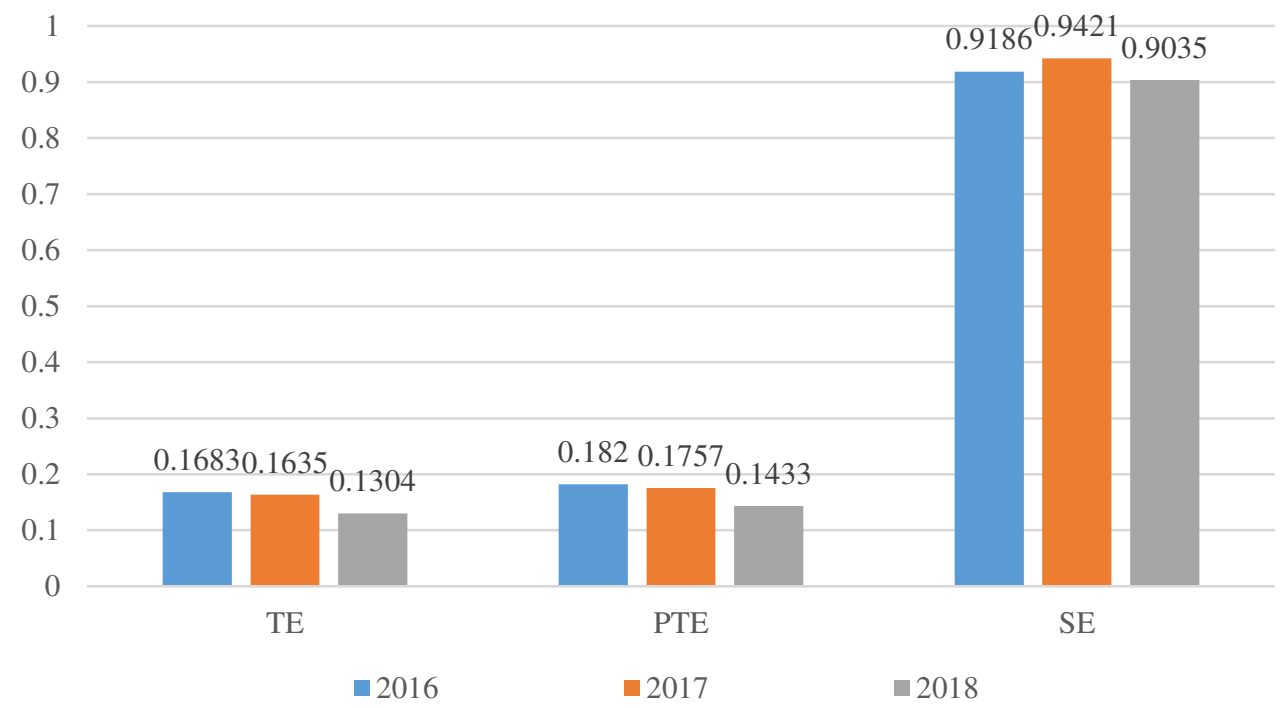

Fig 1. Technical efficiency (TE), pure technical efficiency (PTE) and scale efficiency (SE) of rural medical health centers from 2016 to 2018

The $\alpha$ convergence test results showed that the $\alpha$ values of the RMHCs were $0.7607,0.7897$, and 0.8473 , respectively, for 2016, 2017, and 2018. This shows that 
the RMHCs' efficiency showed a trend of continuous growth and no evident downtrend year on year, indicating no $\alpha$ convergence between the efficiencies of different RMHCs, which means that the efficiency did not decrease during the period 2016-2018. The absolute $\beta$ convergence test results showed that the regression coefficient of $\beta$ is negative and significant at the $1 \%$ level, indicating that the efficiency exhibited absolute $\beta$ convergence, which means that RMHCs with a low initial medical service efficiency had a higher growth rate, whereas RMHCs with a high initial medical service efficiency had a lower growth rate. As a result of this growth gap, the medical service efficiency gap among RMHCs will gradually narrow. In addition, the coefficient in the conditional $\beta$ convergence model is negative and significant at the $1 \%$ level, indicating that the medical service efficiency of RMHCs approaches its steady-state level over time (see Table 2).

Table 2 Convergence of rural medical health centers

\begin{tabular}{cccccc}
\hline & \multicolumn{2}{c}{ Absolute $\beta$ convergence } & & \multicolumn{2}{c}{ Conditional $\beta$ convergence } \\
\cline { 2 - 3 } & coefficient & Std.Err & & coefficient & Std.Err \\
\hline $\ln T E_{i, 0}$ & $-0.0840^{* * *}$ & $(0.0039)$ & $\ln T E_{i, t-1}$ & $-0.1931^{* * *}$ & $(0.0069)$ \\
$\mathrm{F}$ & 450.81 & & $\mathrm{~F}$ & 775.27 & \\
$\mathrm{R}^{2}$ & 0.0853 & & $\mathrm{R}^{2}$ & 0.0743 & \\
\hline
\end{tabular}

\section{Discussion}

This study analyzed the efficiency and convergence of RMHCs in Shanxi Province, China. We found that the RMHCs were facing a low level of medical service efficiency because of insufficient health care resource investment. The convergence results indicated that the medical service efficiency of the RMHCs would improve and approach its homeostatic level if policies can be appropriately implemented.

From the perspective of the government's investment into RMHCs, the number of rural doctors and drug expenditure showed a decreasing trend. Rural doctors 
represent important human resource in RMHCs; unfortunately, the number of rural doctors has been declining in recent years. According to the China Health Statistics Yearbook 2019, the number of rural doctors in China has dropped from 1,031,828 in 2010 to 845,436 in 2018 , a drop of approximately $18 \%$, and there have even been mass resignations of rural doctors ${ }^{[21]}$. The reason is that their income source mainly includes three aspects: basic drugs system subsidy, basic public health services subsidy, and diagnosis and treatment income. The subsidies were the main source of income. However, as RMHCs are at the bottom of the rural medical system, the subsidies were deducted, with little left for rural doctors ${ }^{[22]}$. In addition, rural doctors not only undertake basic medical services for rural residents but also undertake considerable basic public health services. This makes them dissatisfied with the current situation ${ }^{[23]}$. On the other hand, the decrease in the drug expenditure is significantly related to the limited essential medicine varieties. In this situation, patients prefer to go to higher-level hospitals ${ }^{[24]}$.

From the efficiency evaluation results of the RMHCs, the average TE and PTE scores of RMHCs in the past three years were only 0.1541 and 0.1670 , respectively. In particular, in 2018 , nearly $63 \%$ of the TE and PTE scores were below their average level. At the same time, the TE and PTE scores were still decreasing year on year. Based on the previous analysis, it can be concluded that the decrease in the TE and PTE scores year on year is strongly related to the decline in the number of rural doctors and the insufficient input of drug resources. RMHCs should be paid more attention in this regard. Therefore, an effective way to improve the medical service efficiency of RMHCs is to enhance the dynamic management of rural doctors and drug procurement. First, the government should improve the income subsidy mechanism for rural doctors, increase the proportion of national financial support, and reduce the proportion of county-level subsidies to ensure the availability of subsidy funds ${ }^{[25]}$. Second, county, township, and village health service networks should be established to set up a service platform for sharing human resources for healthcare. Third, the medical insurance agency should adjust the list of essential drugs 
dynamically to ensure their adequate supply.

From the efficiency of RMHCs' development trend, the absolute $\beta$ convergence and conditional $\beta$ convergence indicate that health equalization in rural area can be achieved; however, notably, the connotation for promoting the equalization of rural medical and health resources among regions is that rural residents have equal access to medical and health resources when they need to visit hospitals. Therefore, the equalization of regional medical and health resources pursued by the government is not an absolute $\beta$ convergence among regions but a conditional $\beta$ convergence based on certain factors. In other words, when allocating medical and health resources, the government should dynamically manage medical and health resources depending on the level of economic development, and population size and age structure of the rural residents in rural areas, to ensure that the RMHCs reach their own optimal level of medical service efficiency.

\section{Conclusion}

Overall, the medical service efficiency of RMHCs remains low and may gradually decline. The government should promote the efficiency of medical service in RMHCs to reach their own optimal level by formulating corresponding medical and health resource allocation policies.

\section{Limitation}

This paper has several limitations. First, the sample RMHCs were selected from a northern province in China; other provinces were not selected in our study. Second, because of data access permissions, none of the output indicators in the DEA model were medical quality indicators. In the future, scholars can include more provinces in China and choose some medical quality indicators in the DEA model.

\section{Acknowledgements}

Not applicable 


\section{Authors'coutributions}

All authors made significant contributions to this study. Yun Ye conceptualised the study. Li Jing and Wei Lu collected and analysed the data. Yun Ye wrote the first draft of the manuscript. Li Jing,Richard Evans and Xudong Shang interpreted the results and revised the manuscript. Shaoqiong Li,Wei Lu and Xudong Shang critically commented on the paper. The final version submitted for publication was read and approved by all authors.

\section{Funding}

This study was fsupported by the National Social Science Foundation of China (NO.17BZZ026).

\section{Availability of data and materials}

The data sets analysed during this study are available from the corresponding author upon reasonable request.

\section{Ethics approval and consent to participate}

Not Applicable.

\section{Consent for publication}

Not applicable.

\section{Competing interests}

The authors declare that they have no competing interests. 


\section{Reference}

[1] ZHANG T, XU Y, REN J, et al. Inequality in the distribution of health resources and health services in China: hospitals versus primary care institutions[J]. International Journal for Equity in Health, 2017, 16.

[2] LI B, ZHANG L. Complex Networks Theory Building for Integrated Healthcare Research in Rural China[J]. International Journal of Integrated Care, 2018, 17(5):381.

[3] LU W, EVANS R D, ZHANG T, et al. Evaluation of resource utilization efficiency in obstetrics and gynecology units in China: A three-stage data envelopment analysis of the Shanxi province[J]. International Journal of Health Planning and Management, 2020, 35(1):309-317.

[4] ZHONG K, CHEN L, CHENG S, et al. The Efficiency of Primary Health Care Institutions in the Counties of Hunan Province, China: Data from 2009 to 2017[J]. International Journal of Environmental Research and Public Health, 2020, 17(5).

[5] PAUL, MARSCHALLSTEFFEN, FLESSA. Efficiency of primary care in rural Burkina Faso. A two-stage DEA analysis[J]. Health Economics Review, 2011.

[6] KIRIGIA J M, SAMBO L G, RENNER A, et al. Technical efficiency of primary health units in Kailahun and Kenema districts of 
Sierra Leone[J]. International archives of medicine, 2011, 4:15-15.

[7] SINAY T. Productive efficiency of rural health clinics: The Midwest experience[J]. Journal of Rural Health, 2001, 17(3):239-250.

[8] SHERMAN H D. Hospital Efficiency Measurement and Evaluation: Empirical Test of a New Technique[J]. Medical Care, 1984, 22(10):922-938.

[9] GöZDE Y. Health Efficiency Measurement in Turkey By Using Data Envelopment Analysis: A Systematic Review[J]. Ankara Sağlık Bilimleri Dergisi, 2017(1-2-3):49-69.

[10] KIM J, KIM H, LEEM B, et al. Analyzing the National Medical Service Efficiency of OECD Countries Using DEA and Malmquist Productivity Index[J]. Acta Biomaterialia, 2012, 37(4):197-207.

[11] NASIRIPOUR A A, MEHROLHASANI M H. The Efficiency of the Head Offices of Medical-Services Insurance Organization in Iran Using AP-DEA: 2009[J]. Journal of Health Administration (JHA) 2011 Vol. 14 No. 46 pp. Pe39-Pe46, En8, 2011, (46):39-46.

[12] WANG Z. The convergence of health care expenditure in the US States[J]. Health Economics, 2009, 18(1):55-70.

[13] HAPPICH M, VON LENGERKE T. Convergence of life 
expectancy in the European Union: a Markov approach[J]. Applied Economics Letters, 2007, 14(3):175-178.

[14] GAECHTER M, THEURL E. Health status convergence at the local level: empirical evidence from Austria[J]. International Journal for Equity in Health, 2011, 10.

[15] JI Y B, LEE C. Data envelopment analysis[J]. Stata Journal, 2010, 10(2):267-280.

[16] TONE K. A slacks-based measure of super-efficiency in data envelopment analysis[J]. European Journal of Operational Research, 2002, 143(1):32-41.

[17] CHARNES A, CLARK C T, COOPER C W, et al. A DEVELOPMENTAL STUDY OF DATA ENVELOPMENT ANALYSIS IN MEASURING THE EFFICIENCY OF MAINTENANCE UNITS IN THE U.S. AIR FORCES[J]. Annals of Operations Research, 1985, 2(1):95-112.

[18] SALA-I-MARTIN X. The Classical Approach to Convergence Analysis[J]. Economics Working Papers, 1995.

[19] BAUMOL W J. Productivity Growth, Convergence, and Welfare: What the Long-Run Data Show[J]. American Economic Review, 1986, 76(5):1072-1085. 
[20] BARRO R J, SALAIMARTIN X. Economic Growth and Convergence across The United States[J]. Nber Working Papers, 1990.

[21] CHEN ZHONGMIN Y W, JIA HAIYI. Formation Mechanism of the Turnover Intention of $\mathrm{R}$ ural Doctors Under Essentail Medicine System Based on Grounded Theory[J]. Chinese General Practice, 2015, 18(25):3036.

[22] LE, YANG, HONGMAN, et al. Who will protect the health of rural residents in China if village doctors run out?[J]. Australian Journal of Primary Health, 2019.

[23] WANG Q, KONG Y, SUN J, et al. What Are the Challenges Faced by Village Doctors in Provision of Basic Public Health Services in Shandong, China? A Qualitative Study[J]. International Journal of Environmental Research \& Public Health, 2019, 16(14):2519.

[24] Zhou, Su Y, Campbell B , et al. The Financial Impact of the 'Zero-Markup Policy for Essential Drugs' on Patients in County Hospitals in Western Rural China[J]. Plos One, 2015, 10(3):e0121630.

[25] Xiao-Qiang Q , Wen-Qiang Y, Dong-Mei H , et al. Research on the development of rural doctors in the background of the new healthcare reform based on the grounded theory[J]. Chinese Journal of Health Policy, 2016. 
Figures

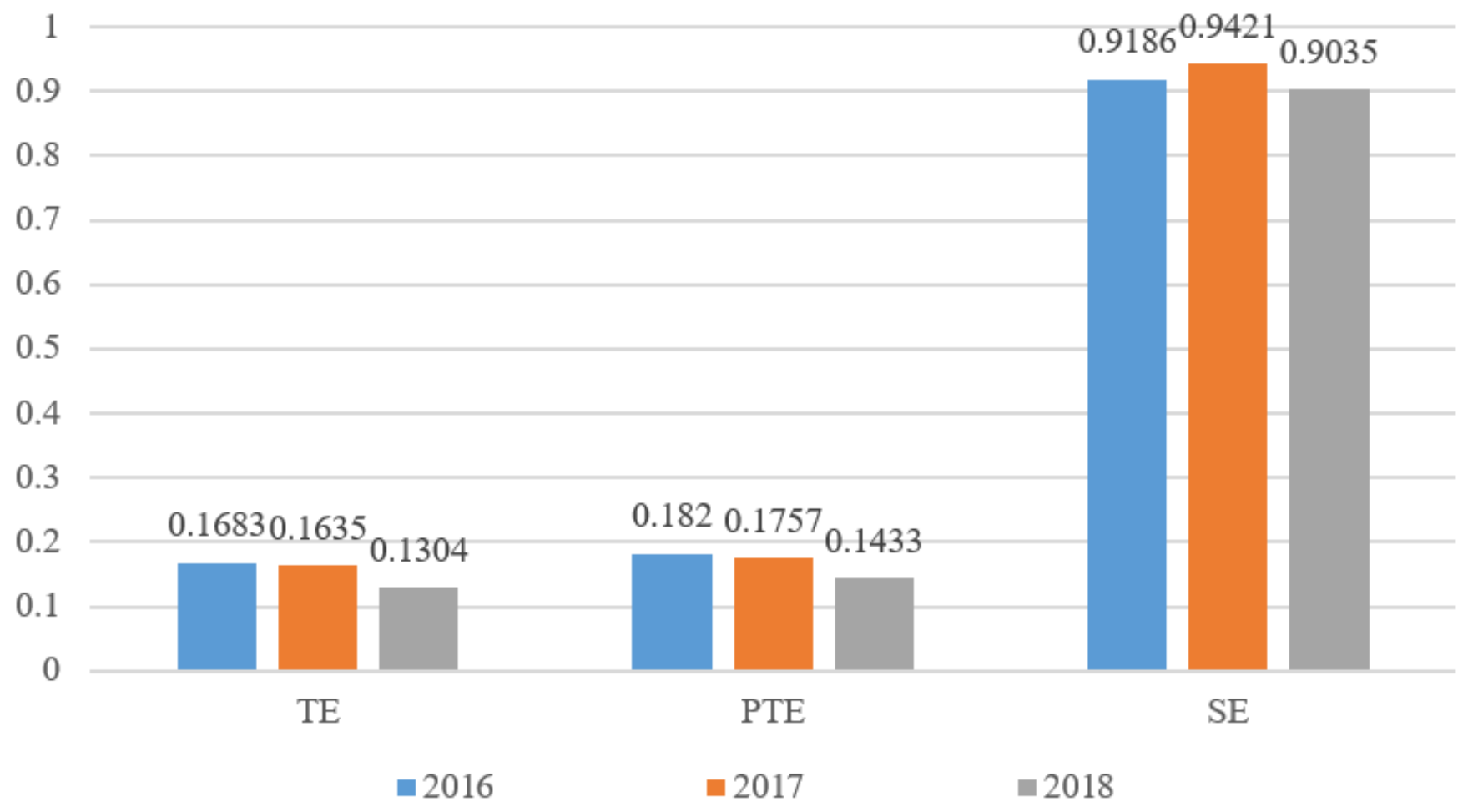

Figure 1

Technical efficiency (TE), pure technical efficiency (PTE) and scale efficiency (SE) of rural medical health centers from 2016 to 2018 